INTERVIEW

For reprint orders, please contact: reprints@futuremedicine.com

\section{Magnetic resonance-guided high-intensity-focused ultrasound for the treatment of cancer-related pain}

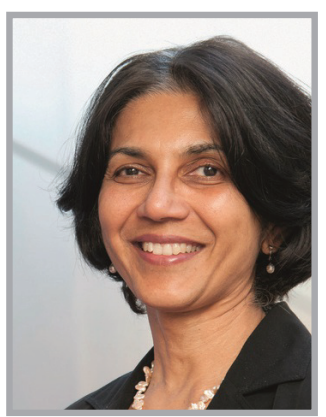

Nandita deSouza* speaks to Nick Ward, commissioning editor: Nandita deSouza speaks about her involvement in the development of high-frequency ultrasound ( $\mathrm{HiFU}$ ) for the treatment of cancer-related pain. Nandita deSouza is a Professor of translational imaging at The Institute of Cancer Research, (ICR, London, UK) and Honorary Consultant at The Royal Marsden Hospital (London, UK). N deSouza is a codirector of the Cancer Research UK Clinical Magnetic Resonance Research Group at the ICR. In this role she coordinates multidisciplinary research projects involving clinical medicine, physics, biochemistry and engineering that are aimed at improving patient care.

Q Please tell our readers a little about your position \& your current research? I am a Professor of translational imaging at the Institute of Cancer Research (ICR) and a Consultant Radiologist at The Royal Marsden Hospital. In my role, I sit at the interface of preclinical and developmental work and the rollout of new technologies to the clinic. My research mainly involves MRI and it is my remit to take new developments in magnetic resonance, or help initiate new developments in MRI, and bring them to fruition in clinical studies. I have been at the ICR and The Royal Marsden for 11 years and in that time, I have been leading the clinical group in the Centre for Cancer Imaging. The group as a whole has a broad remit and includes physicists, chemists and biologists as well as imaging scientists.

\section{Q When did your involvement in the high-frequency ultrasound treatment \& development begin?}

I previously worked at Imperial College (London, UK), and collaborated with Gail ter Haar from the ICR, a renowned figure in the high-frequency ultrasound (HiFU) community. We now work together at the ICR and The Royal Marsden. She is a physicist who has spent many years developing $\mathrm{HiFU}$ and is an international authority on the topic. Our initial work investigated the development of a novel device to deliver HiFU for prostate treatments under MRI guidance. At this point, it was very much at the bench level. Then, when I moved to The Royal Marsden, I kept up my interest in HiFU, and when the opportunity arose for us to take advantage of a Philips product that could actually deliver HiFU under MRI guidance, it was a great opportunity. The Focused Ultrasound Foundation really helped to facilitate this by giving us a grant and then Philips was able to loan us the equipment to enable the whole program to take off. With the Focused Ultrasound Foundation funding and the Philips equipment, we were able to bring HiFU to the clinic.

\section{Q What are the other clinical applications of HiFU?}

An application of HiFU performed under ultrasound rather than MRI guidance is in treating prostate cancer. However, this uses a different device that is a rectal probe, needed for treating localized prostate cancers. One negative of this application is that it lacks the

*The Institute of Cancer Research, Cotswold Road, Sutton, Surrey, SM2 5NG, UK; Nandita.Desouza@icr.ac.uk

\section{Pain Management}

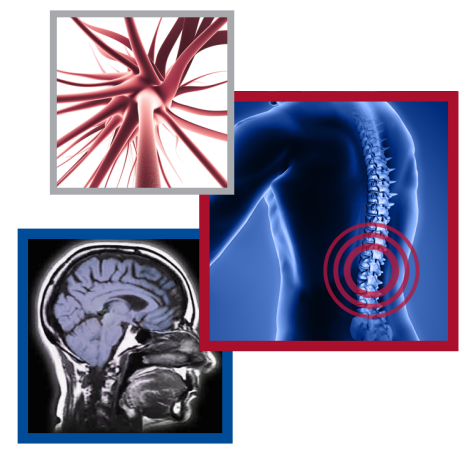

\section{KEYWORDS}

- bone metastasis • cancer

- high-intensity-focused ultrasound

- magnetic resonance $\bullet$ pain 
temperature monitoring capability available with MRI. MRI-guided HiFU treatment made its name by treating fibroids in the uterus. These are not malignant cancers and HiFU has been used to ablate these effectively, burning them away and in doing so removing the symptoms of heavy, painful periods. For this particular application, $\mathrm{HiFU}$ is now a recognized treatment and there are many thousands of patients who have benefited from this treatment worldwide. The first application of MRI guided HiFU in cancer treatment is for the treatment of secondary bone tumors that are painful. This is currently the focus of the clinical trial in which we are participating.

Q How the patients who have undergone HiFU treatment for cancer pain benefited?

$\mathrm{HiFU}$ is a very precise treatment, similar to focusing the sun's rays through a magnifying glass. Instead of focusing light, ultrasound at high power is focused to a very precise spot; at the focal spot a very precise burn is produced. The objective with the pain relief for secondary bone cancers is to deliver these precise burns along the surface of the bone where the pain is experienced. No one has definitively demonstrated the mechanism for the observed pain relief but it is believed that the nerve endings supplying the bone surface are burnt away, so that the sensation of pain is no longer experienced. In treating cancer-related pain, it is common that patients have secondary deposits in the bone all over the body and it is not possible to treat all those lesions. Therefore, the purpose of using $\mathrm{HiFU}$ is to treat a specific painful lesion at the point of pain in order to achieve pain relief. For example, one of our patients had shoulder pain and consequently required assistance with everyday tasks, such as getting dressed, opening cupboards and walking the dog. With HiFU treatment to a specific point of pain she is now able to do these tasks. Another example is a patient who had pain in the lower back that was worse at pressure point when he was lying down and so kept him awake all night. After treatment with $\mathrm{HiFU}$, he is now able to get some sleep at night as his pain decreased. In both these cases we have not cured the cancer, but the decrease in pain hugely improved the patients' quality of life.

\section{Q What are the observed side effects with} HiFU treatment for cancer-related pain? Currently, we have treated five patients with $\mathrm{HiFU}$ and none of these patients have experienced any side effects. The most commonly reported side effects are burns to the skin over the site that has been treated, where the energy deposited is on the skin surface and this can result in a redness of the skin or in the worst case scenario, a burn. There have been reported side effects in scientific publications, but we have been fortunate enough to not to experience them in our patients so far.

\section{Q How does the real-time visualization} \& precise nature of HiFU treatment compare with conventional treatments for cancer pain? Conventionally, patients who present with pain arising due to cancer within the bone, are treated with drugs (tablets) to achieve pain relief. Specific treatment to a local site requires radiotherapy. The resolution of images used to deliver radiotherapy is low and the tumor is not visualized during treatment. Furthermore, the radiotherapy is often delivered over multiple sessions. In comparison, HiFU treatment is a one-off treatment and when delivered under MRI, detailed images of the area being treated are available. During the delivery of the treatment, the treatment area is repeatedly imaged to obtain a real-time 'temperature map'. This 'temperature map' is then overlaid on an image of the treatment area and provides real-time information on how much the temperature of the tissue has been raised. Delivery of each burst of HiFU lasts 12-15 s, during which the rise in temperature and where precisely this rise in temperature is occurring can be mapped. When the treatment burst is complete, a repeat scan allows visualization of what changes have occurred in the tissue as a result of the treatment. Obviously this is quite different to how radiotherapy is delivered. HiFU is not a replacement for radiotherapy, which is an extremely effective treatment for cancer. Given its precision, HiFU is more ideally suited to treating areas the size of a pencil tip rather than for treating large tumors several centimeters in diameter. In the latter case, many overlapping burns are required to achieve an effective treatment, which could take hours.

\section{Q What are the next steps in the clinical trials to develop this treatment?}

All the current clinical trials are focused on the palliation of bone pain and while this is a very laudable aim, I feel there are many other applications in cancer treatment that would benefit from MRI guided HiFU. One aspect could be 
to develop MRI guided HiFU to destroy local tumors and this is really where the imaging comes in. It is not just about the HiFU, but also about being sure that you have treated all of the tumor and the only way to be sure of this is to image it. Take the example of a $2 \mathrm{~cm}$ breast tumor, which could be treated with HiFU. The reason these tumors are currently taken out surgically is because doctors need to be sure that the tumor has been taken out in its entirety. The only way you can know this for sure is by examining it under the microscope. If the area could be imaged to show whether or not the tumor has been left behind after HiFU reliably - which also depends on increasing the accuracy of the imaging technique - then this would be a very powerful reason for using MRI guided $\mathrm{HiFU}$ to treat local tumors. That is something we aspire to. In other clinical scenarios, there are tumors which have recurred after surgery, they have had radiotherapy, and chemotherapy is failing to control tumor growth. In such cases, I think that HiFU will play a very important role because it does not have the same degree of toxicity and side effects as further drug treatments and radiotherapy. Because HiFU is so precise, and the warming of tissues through which the beam passes away from the focus is insufficient to cause harm to surrounding tissue, HiFU could be a very important treatment modality for patients where all other treatment options have been exhausted. I think that collaboration between the different research groups will facilitate more studies and ultimately develop solutions involving HiFU sooner.

\section{Q What are the cost implications of HiFU compared with conventional treatments for cancer-related pain?}

It is early days in the development of HiFU and at this point in time, I would say that it is likely that the cost is very comparable to existing treatments. We have the advantage that $\mathrm{HiFU}$ is a one-off treatment and there is no toxicity to manage, so these things will come out in favor economically. However, at present, the treatment is quite expensive to implement due to the whole workflow taking much longer to implement, as we are not ready to deal with a high throughput of patients. Ultimately, the costs will come down and it will become cheaper. It is not going to be an exclusive treatment that is unaffordable; however, I do see it being limited to cancer treatment centers and it would have to be properly managed by specially trained staff. There is potential scope to develop much smaller, portable devices that could be used in clinics in the community, but that is a long way off.

\section{Q How important are industry partners for the development of HiFU?}

I think industrial partners are vital in the development of technology such as this. It is very much a two-way process, they have got a very good technology that they have developed and understood, and we have a set of clinical applications and needs. Each party (academic and industrial) needs the expertise of the other. Industry partners need to know the specifications required from ourselves, as much as we need to know from them is what this device can do and how we can use it to our advantage. This back and forth with industry partners is vital and this is why we have a very good working relationship with Philips and are able to discuss such matters.

\section{Q What are the main obstacles that HiFU} needs to overcome before it can be used as widely available treatment modality for cancer pain?

It is not an obstacle as such, but in the first instance, it is familiarity and proving that the treatment is effective and that will come with the clinical trial reports. There are currently seven clinical trials ongoing involving MRI guided HiFU. If the final reports indicate that the treatment is effective and the complication rate is low, that is the first obstacle overcome. After this, treatment protocols need to be developed and treatment delivery optimized while managing clinical expectations. Then there is the economic issue of where the equipment is available and also a training issue of who has the expertise to use the equipment. I think that once the trials report, there will be the demand for the technique, manufacturers will get orders and then training workshops would have to be organized so that individuals experienced in using HiFU can pass on the knowledge to colleagues.

\section{Q How do you see this field developing in} the next 5-10 years?

There are currently researchers investigating the use of HiFU to enhance drug delivery. For example, if you can deliver a drug contained in 
a 'package' or coating can be administered that requires a burst of thermal energy to remove the 'package', so that a high concentration of active drug can be achieved at the site of a tumor without inflicting the side effects of the drug on the rest of the body. Another aspect will be combining MRI-guided HiFU with MRI-guided radiotherapy. Areas of some tumors are poorly supplied by oxygen, limiting the effectiveness of the radiotherapy. This is where the imaging is important: imaging the tumor and locating the area that is less responsive to radiotherapy would enable this small area to be treated with HiFU before or after the rest of the tumor is treated with radiotherapy, combining the two treatments for a better outcome. This strategy can be applied to drug treatments as well as radiotherapy.

\section{Disclaimer}

The opinions expressed in this interview are those of the interviewees and do not necessarily reflect the views of Future Medicine Ltd.

Financial \& competing interests disclosure

$N$ deSouza receives support from Cancer Research $U K$ who fund a 5-year Imaging Centre grant at ICR, the NIHR who fund a Clinical Research Facility in Imaging at the Royal Marsden Hospital, The EU iMi QuIC ConCePT project and the Focused Ultrasound Foundation who have established a Centre of Excellence at ICR. N deSouza has no other relevant affiliations or financial involvement with any organization or entity with a financial interest in or financial conflict with the subject matter or materials discussed in the manuscript apart from those disclosed.

No writing assistance was utilized in the production of this manuscript. 